\title{
Computed Tomography/Diffusion Weighted Magnetic Resonance Imaging Fogging Effect in the Subacute Stage of Ischemic Cerebral Infarct: A Report of Three Cases
}

\author{
Geo Seong Park, Hyoung-Gyu Jang, Jung-Soo Park, Jong-Myong Lee, Eun Jeong Koh, Ha-Young Choi \\ Department of Neurosurgery, Research Institute of Clinical Medicine of Chonbuk National University, Biomedical Research Institute \\ of Chonbuk National University Hospital, Jeonju, Republic of Korea \\ Corresponding author: \\ Hyoung-Gyu Jang \\ Department of Neurosurgery, Chonbuk \\ National University Hospital, Chonbuk \\ National University, 20, Geonjiro Deokjin-gu, \\ Jeonju 54907, Republic of Korea \\ Tel: $+82-63-250-1870$ \\ Fax: $+82-63-277-3273$ \\ E-mail: hgjang121180@naver.com \\ Received: May 2, 2021 \\ Acute cerebral infarctions usually demonstrate hypodensity on non-contrast computed tomo- \\ graphy (CT). However, in some cases, cerebral infarctions may appear to be isodense on ima- \\ ging conducted during the subacute stage (2-3 weeks after onset). This phenomenon was \\ previously called the fogging effect and has also been reported in magnetic resonance ima- \\ ging. It is generally reported at T2 image, but can also be observed in diffusion-weighted image \\ (DWI). We report three cases of the fogging effect demonstrated on CT and DWI that was \\ conducted in the subacute stage of ischemic cerebral infarcts. The fogging effect can result in \\ incorrect judgment during the diagnosis and treatment of cerebral infarction patients in the \\ subacute stage. Therefore, it is important that clinicians anticipate this occurrence and necessi- \\ tates the development of better detection methodologies.
}

Revised: May 27, 2021

Accepted: May 28, 2021
Key Words: Cerebral infarction; Magnetic resonance imaging; Stroke; Tomography, X-ray computed

\section{INTRODUCTION}

Hypodensity on non-contrast computed tomography (CT) is typically observed 12 to $24 \mathrm{hr}$ following acute cerebral infarctions ${ }^{3)}$. However, in some cases, cerebral infarctions may briefly disappear on imaging 2 to 3 weeks after onset. Becker et al. ${ }^{2)}$ first described this phenomenon using the term "fogging effect" in 1979 and similar occurrences have also been reported in magnetic resonance (MR) imaging (MRI). It is generally reported at T2 image, but can also be observed in diffusion-weighted image $(\mathrm{DW})^{1,2)}$.

Studies have reported the occurrence of the fogging effect to be anywhere between $10 \%$ to $54 \%$ of reported cerebral infarction cases $4,6,8,9)$. This wide range of prevalence rates may be partly attributed to misinterpretation of the lack of radiological findings by radiologists or physicians despite clinically suspected cerebral infarction. Therefore, it is necessary to understand the fogging effect to facilitate accurate interpretation of CT or MRI data and appropriate treatment. Herein, we report three cases illustrative of the fogging effect on CT and MRI scans obtained during the subacute stage of cerebral infarction.

\section{CASE REPORT}

\section{Case 1}

A 12-year-old boy presented to the emergency department at our institution with a loss of consciousness after a pedestrian accident. The patient's Glasgow Coma Scale score at this time was 3 points.

No evidence of intracranial hemorrhage or cerebral infarction was detected on the initial non-contrast head CT (Fig. 1A). On the second day of hospitalization, the patient's state of consciousness progressed to a stupor and the follow-up non-contrast head CT demonstrated hypodensity in both occipital lobes (Fig. 1B). A DW obtained on the same day demonstrated high signal intensity corresponding to the head CT findings (Fig. 1C). By day 18 post-injury, the patient's consciousness improved to a drowsy state. Isodensity was noted on a follow-up non-contrast head CT scan in the previously hypodense area (Fig. 1D). Similarly, repeat DW did not demonstrate any high signal intensity (Fig. 1E). By day 40, the hypodensity was redemonstrated (Fig. 1F). The patient was subsequently transferred to another hospital for rehabilitation and remained in a drowsy state of consciousness at discharge.

\section{Case 2}

A 61-year-old woman with a history of hypertension and hyperlipidemia presented to the emergency department with a sudden onset headache. Though alert at the visit, the patient developed a seizure for which she was sedated.

The initial non-contrast head CT scan revealed a subarachnoid hemorrhage and a subsequent cerebral digital subtraction angiography (DSA) indicated a saccular aneurysm of the left anterior inferior cerebellar artery (AICA) (Fig. 2A, B). An emergency endovascular 

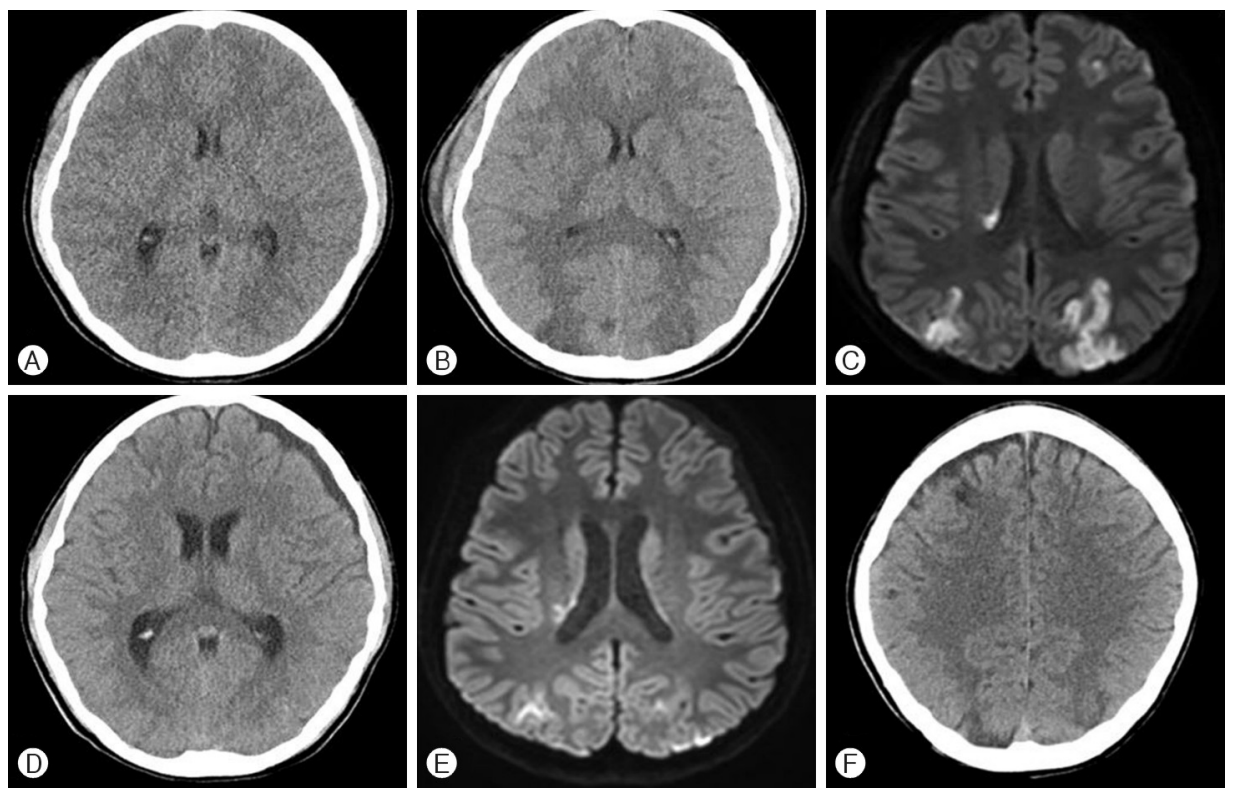

Fig. 1. Imaging studies for Case 1. (A) Day 1, non-contrast computed tomography (CT) shows no specific lesion. (B) Day 2, non-contrast CT shows hypodensity in both occipital lobes. (C) Day 2, diffusion-weighted image (DM) shows high signal intensity. (D) Day 18, non-contrast CT shows isodensity. (E) Day 18, DM changed to isosignal intensity. (F) Day 40, noncontrast CT changed to hypodensity.
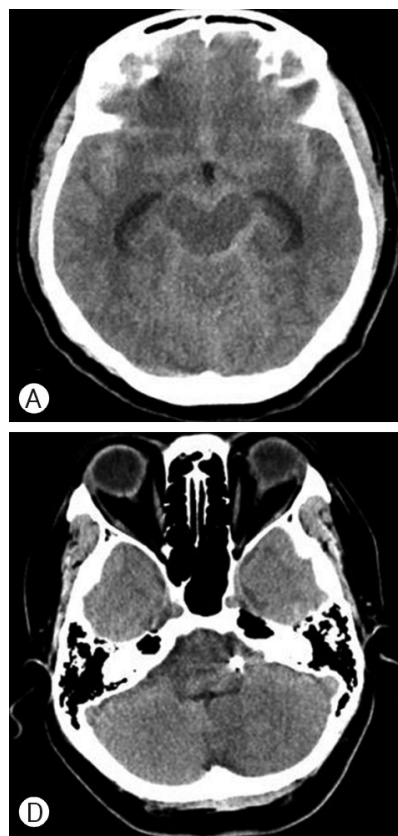
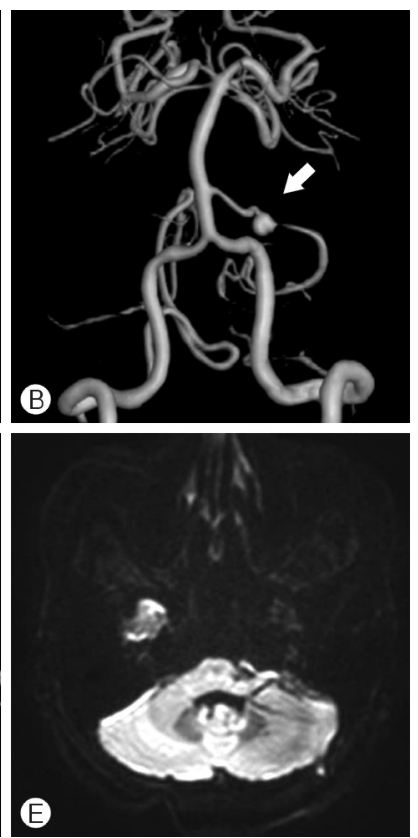
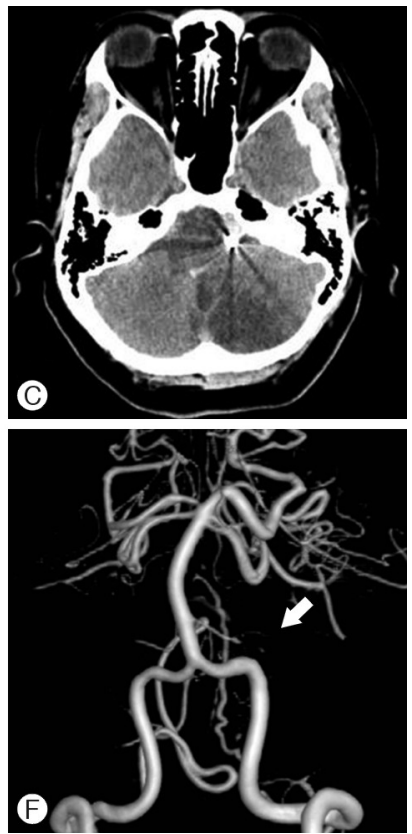

Fig. 2. Imaging studies for Case 2. (A, B) Day 1, non-contrast computed tomography (CT) \& data acquisition system (DAS) demonstrated subarachnoid hemorrhage due to ruptured left anterior inferior cerebellar artery (ACA) (C) Day 4, non-contrast CT shows hypodensity in the left AICA territory. (D) Day 14, non-contrast CT changed to isodensity. (E) Day 20, diffusionweighted image confirmed to isosignal intensity. (F) After 1 year, DAS demonstrated blocked AICA distal flow. internal trapping of the aneurysm was performed using coils, resulting in distal AICA flow blockage.

The hypodensity lesion was observed on a non-contrast head $\mathrm{CT}$ in the left AICA territory of cerebellar cortex 4 days post-admission (Fig. 2C). By day 14, these changes were not demonstrated (Fig. 2D). Similar isointense findings were noted on a DW conducted on day 20 of hospitalization (Fig. 2E). The patient's consciousness and symptoms, including the headache, improved and she was discharged without any complications. A year later, distal flow of the AICA blockage was demonstrated again via a DSA exam (Fig. 2F).

\section{Case 3}

A 25-year-old woman who presented with mental deterioration and left-sided weakness was admitted to the emergency room in our hospital. Initial non-contrast head CT and DW scans revealed a cerebral infarction in the right temporoparietal lobe (Fig. 3A, B) and subsequent MR angiography findings indicated moyamoya disease. The patient underwent conservative management of cerebral infarction. DW conducted on day 10 following hospitalization showed isosignal intensity in the right temporoparietal region com- 

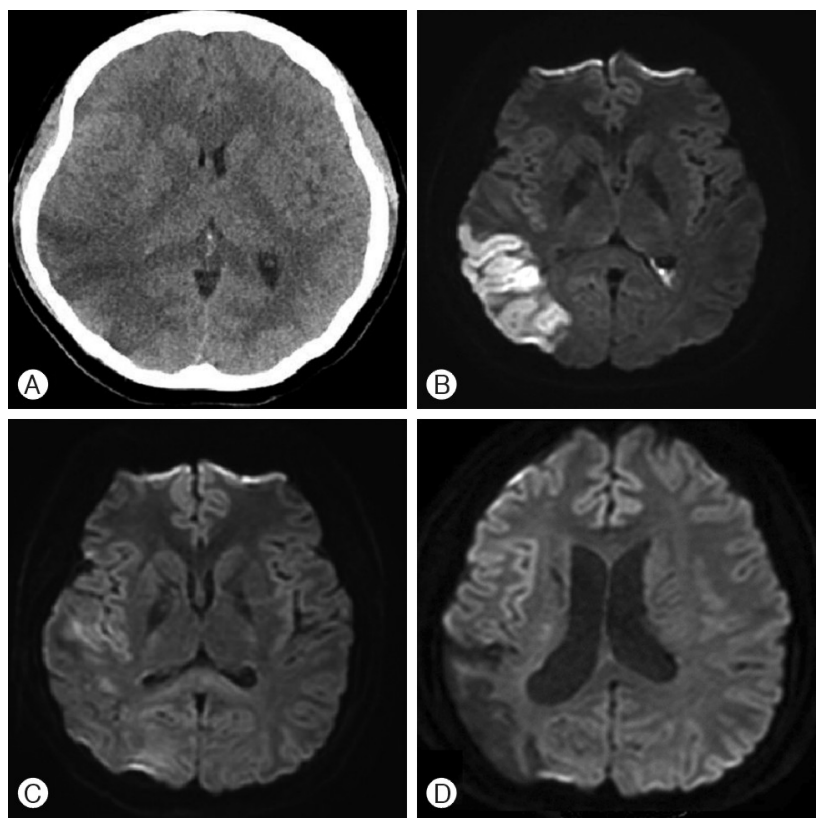

Fig. 3. Imaging studies for Case 3. (A) Day 1, non-contrast computed tomography shows hypodensity in the right temporoparietal lobe. (B) Day 1, diffusion-weighted image (DWI) shows high signal intensity. (C) Day 10, DWl changed to isosignal intensity. (D) After 4 months, DWl shows low signal intensity.

pared to the DWI taken at admission (Fig. 3C). DW performed in the outpatient department 4 months after discharge demonstrated low signal intensity in the right temporoparietal lobe (Fig. 3D).

\section{DISCUSSION}

Hypodensity on non-contrast CT is typically observed 12 to 24 hr following acute cerebral infarctions ${ }^{3}$. However, in rare cases, cerebral infarctions may briefly disappear on imaging 2 to 3 weeks after onset, at the subacute stage of the disease process. Becker et al. ${ }^{2)}$ first described this phenomenon using the term "fogging effect" in 1979 and similar occurrences have also been reported in MRl. It is generally reported at T2 image, but can also be observed in $\mathrm{DW}^{1,2)}$.

Becker et al. ${ }^{2)}$ correlated CT findings to the pathological findings of Spatz's classification ${ }^{7}$. According to this, the fogging effect appears in the resorption stage ${ }^{2,7}$ and is thought to be the result of lipid-laden macrophage invasion, proliferation of capillaries, extravasation of blood cells, and decrease in bulk water in the infarcted area. Since water levels and cellularity in the infarct area can return to near-normal levels in the subacute stage of cerebral infarction, the fogging effect occurs most frequently during this period ${ }^{2,5,7)}$.

When retrospectively reviewed, the three cases reported here conspicuously demonstrate the fogging effect on CT images and
DW. As commonly occurs in clinical practice, we did not initially consider the fogging effect due to lack of awareness, and instead assumed that the disappearance of findings evidenced improvements, not only in terms of imaging but also clinically.

Therefore, it is important to be aware of the possibility of unusual changes in these imaging studies, and care should be taken not to unnecessarily discontinue or alter treatments due to temporary improvements visible on the images. In addition, continued research on imaging methods and interpretations of cerebral infarction in subacute stage might be needed.

\section{CONCLUSION}

In summary, the fogging effect is an unusual presentation in an imaging study that can lead to incorrect judgment in diagnosis and treatment in cerebral infarction patients during the subacute stage. Therefore, possible unusual changes in these imaging studies must be aware for continuous and proper treatment of patients.

\section{CONFLICTS OF INTEREST}

No potential conflict of interest relevant to this article was reported.

\section{REFERENCES}

1. Asato R, Okumura R, Konishi J: "Fogging effect" in MR of cerebral infarct. J Comput Assist Tomogr 15:160-162, 1991

2. Becker H, Desch H, Hacker H, Pencz A: CT fogging effect with ischemic cerebral infarcts. Neuroradiology 18:185-192, 1979

3. Drayer BP, Dujovny M, Boehnke M, Wolfson SK, Jr., Barrionuevo PJ, Cook EE, et al.: The capacity for computed tomography diagnosis of cerebral infarction. An experimental study in the nonhuman primate. Radiology 125:393-402, 1977

4. Masdeu JC, Azar-Kia B, Rubino FA: Evaluation of recent cerebral infarction by computerized tomography. Arch Neurol 34: 417-421, 1977

5. Scuotto A, Cappabianca S, Melone MB, Puoti G: MRI "fogging" in cerebellar ischaemia: case report. Neuroradiology 39:785787, 1997

6. Skriver EB, Olsen TS: Transient disappearance of cerebral infarcts on CT scan, the so-called fogging effect. Neuroradiology 22:61-65, 1981

7. Spatz H: Pathologische anatomie der kreislaufstörungen des gehirns. Z Gesamte Neurol Psychiatr 167:301-351, 1939

8. Wing SD, Norman D, Pollock JA, Newton TH: Contrast enhan cement of cerebral infarcts in computed tomography. Radiology 121:89-92, 1976

9. Yock DH, Jr., Marshall WH, Jr.: Recent ischemic brain infarcts at computed tomography: appearances pre- and postcontrast infusion. Radiology 117:599-608, 1975 